\title{
Analysis of Plant Diseases with Detection using Image Processing Methods
}

\author{
Yatendra Kashyap \\ Assistant Professor \\ Dept, of Computer Science \& \\ Engineering \\ Corporate Institute of science \\ \& Technology,Bhopal,India
}

\author{
Tanuja Sharma \\ Scholar \\ Dept, of Computer Science \& \\ Engineering \\ Corporate Institute of science \\ \& Technology,Bhopal,India
}

\author{
Syed Shahnawaz Ali, PhD \\ Associate Professor \\ Dept, of Basic Science \\ Corporate Institute of science \\ \& Technology,Bhopal,India
}

\begin{abstract}
Crop production is one of the major sources of earning and more than half of our population depends on agriculture for livelihood. Due to the factors like diseases, pest attack and sudden change in the weather condition, the productivity of the crop decreases. Traditional method of checking diseases in plants is through visualization but this method is not so relevant in detecting the diseases associated with plants. So we can provide a better alternative, fast and accurate detection by using image processing techniques which can be more reliable than some other old methods. Through this paper we proposed a methodology for the analysis and detection of plant diseases using digital image processing techniques. Because the fungus and bacteria kills the soya plant foliar and it spread in air and can also infect other plants also. So a close monitoring is required but as a human it is not possible to monitor the large area of land where the crop grows that problem is resolved by our proposed system
\end{abstract}

\section{Keywords}

K-Mean Clustering, Segmentation, Disease Detection, Image processing, Matlab Image processing

\section{INTRODUCTION}

In India about $70 \%$ of population depends on agriculture. Farmers have large choice to select from the diversity of crop. The cultivation technique for good yield should be technical. Monitoring of health of the crop is essential to have a good yield. The diagnostician should have very good detection skills. There can be pests which can affect crop. Each reason has its own importance like proper measures should be taken to control pests and to minimize the loss. The misidentification of pest can lead to the inappropriate measures such as ineffective pesticides which only lead to the waste of time, money and field effort. This can also lead to serious problems in crop.. There can be a case of multiple casual factors also and without knowing the actual truth of disease and its agents, control measures cannot be effective. Therefore proper diagnosis of disease is compulsory. The Image Pre- processing, image segmentation, Feature extraction and classification. This paper provides brief description on this method. For the testing purpose, sample of infected leaf is needed whose image is to be further processed. $\mathrm{K}$ - mean clustering algorithm is used to do segmentation of leaf to the infected. Due to fungus diseases the calculated production losses of US\$55 million each year in India and Southeast Asia [19,6]. For doing so, a large team of experts as well as continuous monitoring is required, which is not practically possible. Another reason is farmers do not have proper facilities or even idea that they can contact to experts. Due to which consulting experts even cost high as well as time consuming too. In such conditions, the proposed methods prove to be beneficial in monitoring large fields of crops with cheaper rate. So this automatic detection will take less time and become more accurate in detection of plant diseases like brown and yellow spots, early and late scorch, and others are fungal, viral and bacterial diseases[6].

\section{LITERATURE REVIEW}

Existing methods for detection of plant diseases and related works:

Plant leaves diseases, its detection and diagnostic method is a scientific method. Digital image processing is a technique which is used and implemented in detection of diseases in pants.The image pre-processing is used to get clear, noiseless enhanced leaves images. These enhanced images are used to leaves diseases detection and its analysis. Various types of images are used in image pre-processing. Generally, plant leaves image color and texture is an unique features, which are used to detect and analyze the diseases. This paper considers Grayscale and RGB images of infected plant leaf detection and their analysis gives the solution which is suited for the disease occurrence.

Various approaches for detection of diseases in plant leaves were discussed in earlier papers .they are as follows:

Dubey $S$ at el \& ravi c: The approach they discussed was only for apple fruit it cannot be extended to other fruits or plants. They proposed approach that composed of three steps such as segmentation, feature extraction and classification. For the image segmentation, they used K-mean clustering technique [1] [9].

S. Arivazhagan: They introduced fuzzy curves and fuzzy surfaces. The features which are extracted from fuzzy selection approach are used for diagnosing and identifying diseases, it removes the dependent features of image so as to reduce the number of features for classification [16].

Rabia Masood developed Genetic algorithm based system whose Disease detection efficiency is about 93\%. Cotton leaves were used for experimentation. Piyush Chaudhary proposed an algorithm for disease spot segmentation using $\mathrm{K}$ meanimage processing and svm.The experiment was carried for three color model i.e. CIELAB, HSI and $\mathrm{YCbCr}$ with conclusion that CIELAB model works better for segmentation of the disease of the leaf using Otsu segmentation[14]

Limitation of existing work:

- The implementation still lacks in accuracy of result in some cases. More optimization is needed.

- Priori information is needed for segmentation. 
- Database extension is needed in order to reach the more accuracy.

- Very few diseases have been covered. So, work needs to be extended to cover more diseases.

- The possible reasons that can lead to misclassifications can be as follows: disease symptoms varies from one plant to another, features optimization is needed, more training samples are needed in order to cover more cases and to predict the disease more accurately.

\section{PROPOSED METHODOLOGY}

The methodological analysis of diagnosing the soybean diseases is shown in the Fig. 1.This process include various tasks, such as image acquisition, image pre-processing, image segmentation, shape feature extraction and soybean disease classification based on lesion type of two most serious disease of soya plant

\subsection{Image Acquisition}

In this process, first we obtain the soybean leaf images. The RGB color digital images of soybean are clicked by using a micromax mobile model canvas 2.2 digital camera, with the pixel resolution $1600 \times 1200$. The digitized images are about $125 \mathrm{~KB}$ size each. There have captured about 100 data samples in the soybean field near vidisha district India. It consists of soybean diseases, namely soybean brown spot and frog eye as shown in Fig. 1. Images are saved and stored in JPG format.

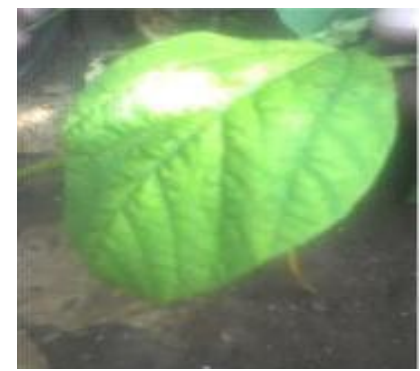

3.2 Image Segmentation and Pre-Processing This is the main task in which pre-processing and segmentation of the soybean image before the image is used for the next process. The main aim of this procedure is to obtain the binary image with noise free. In order to accomplish more exactness, appropriate, interpret should be obtained. The RGB image is converted into background separated image with the help of morphological operation. When we found the background separated image we apply the threshold method [15], to find out the infected region

\subsection{K-mean clustering algorithm}

$\mathrm{k}$-mean is generally used to determine the natural grouping of pixel present in an image it is simple and fast approach so it generally attracts the users .Clustering algorithm is used for the forming of a vector space the objects are clustered around the centeroids

$\mathrm{k}=$ number of clusters that is $\mathrm{si}, \mathrm{i}=1,2,3 \ldots \ldots \ldots \mathrm{k}$ and $\mathrm{u}$ is the mean point or centroid of all points

This algorithm requires colour image as input following steps should be carried out while [performing the k-mean clustering algorithm -

Stage 1: First it read the Original Image

Stage 2: Conversion of Original Image from RGB Colour Space to L*a*b* Colour Space

Stage 3: Classification of the Colour segments in ' $\mathrm{a}^{*} \mathrm{~b}^{*}$ Space by use of K-Means Clustering

Stage 4: Then label the each pixel in the Image from the obtained results of $\mathrm{K}-$ means clustering.

Stage 5: Making of Images that Segment the Hue (H) \& E Image by Colour.

Stage 6: Then Nuclei get segmented into an individual Image

Fig.1 Image captured from mobile

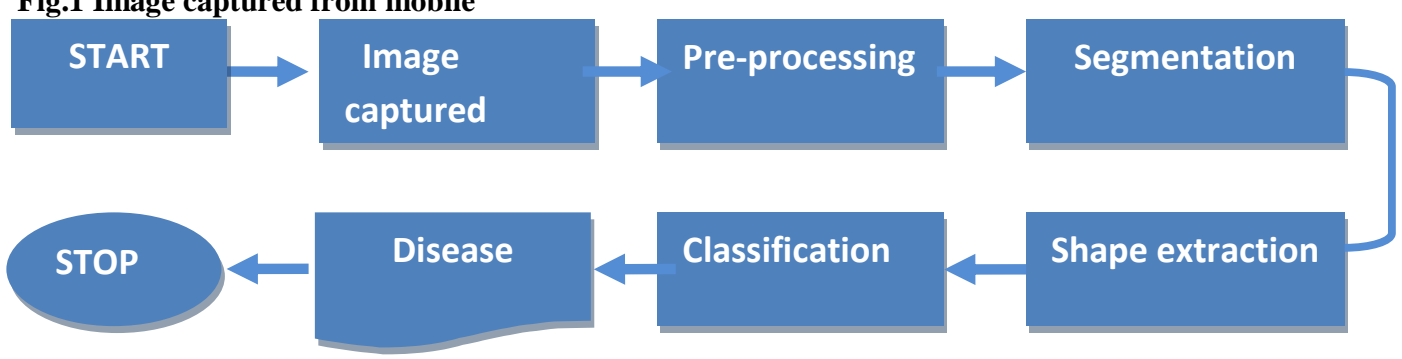

Fig.2 Diagrammatic representation of proposed work

Methodology: There are five main steps for the detection of plant disease -

1. Image acquisition

2. Image pre-processing

3. Image segmentation

4. Feature extraction 


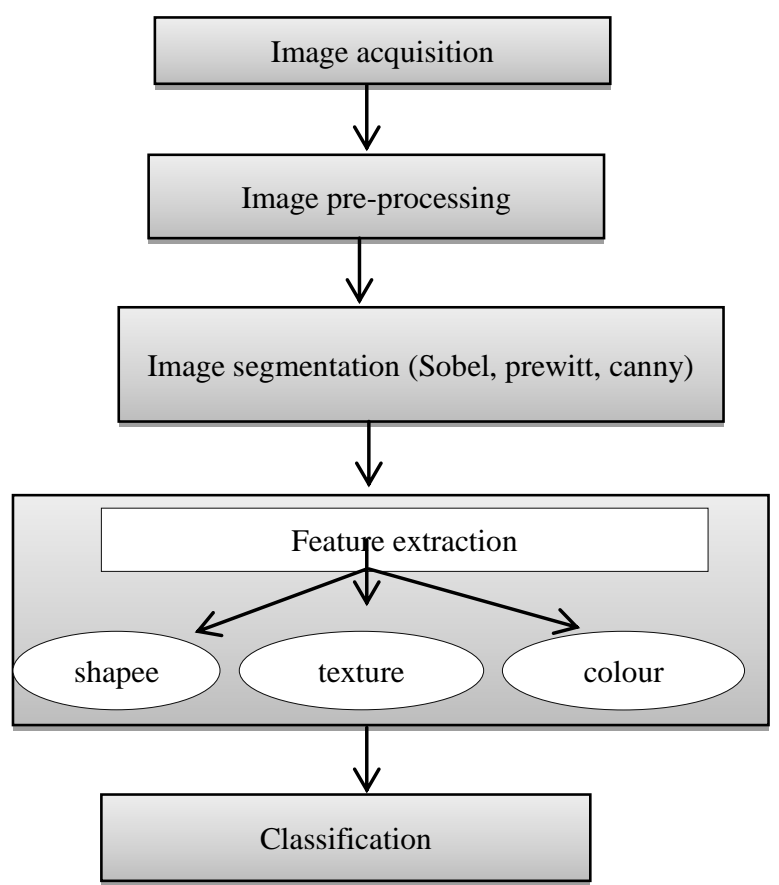

The processing scheme consists of image acquisition through digital camera or scanner, image pre-processing includes image enhancement, and image segmentation where the affected and useful area are segmented, feature extraction and classification which classify disease shows the framework of system.

Image acquisition: it is the first step. The image can be from digital camera or it can be compared with the image available on the internet. If the image is to be taken the following points should be kept in the mind that are the background should be flat and white, optical axis of digital camera should be perpendicular. The image resolution should be minimum $516 * 516$ pixels.

Image pre-processing: this method is also known as image restoring. It enhances the feature of image. It is done to convert the image in appropriate form for testing. this method is also used to separate the foreground and background image. Image pre-processing includes many processes:-

1) Filter image

2) Crop image

3) Resize image

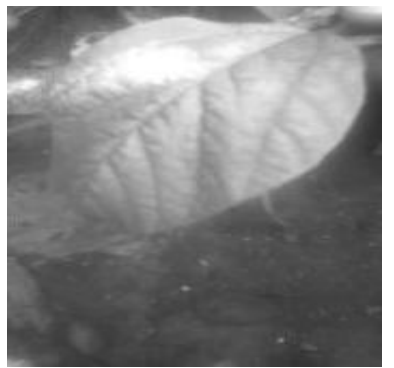

Fig.3 image filtering by mean filter

Image segmentation: segmentation is the method by which we divide leaf into the parts for further studies. The subdivision can be continued until we get the desired result. When we reassemble the segmented parts we should get the original image. in this article we used the k-mean clustering algorithm. There are so many edge detecting algorithms are like sobel,canny ,prewitt,Robert,Log and all these edge detection methods have some characteristics that bifurcate them but in our proposed system we found that canny edge detection menthod give better result as compared with other edge detection algorithms.
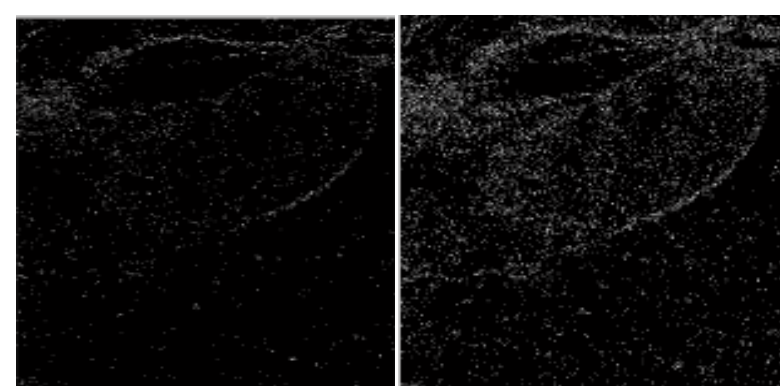

Fig 4.Binary Image

Fig.5 sobel operator (Th-0.09)

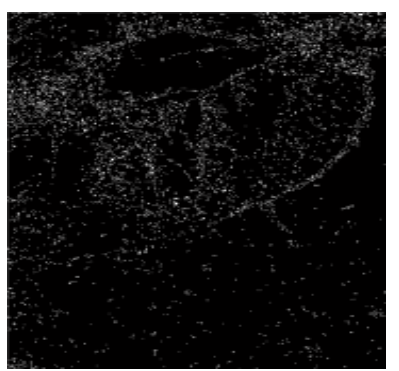

Fig 6. Canny operator (Th-0.09)

Feature extraction: its main purpose is to take the extracted feature, for the meaning of image. It includes colour, shape and texture. Texture is the most important feature as per the researcher, targeting plant leaf structure. Shape is the visual feature which is classified as Boundary based or Region based representation.Colour tells us about brightness and intensity, its degree of purity. By comparing the classification results of ANN and Fuzzy Logic Technique. These analyzes, which system is better in sense of Accuracy, Speed, User friendly, easily adaptable topology of the network changes.[21][22]

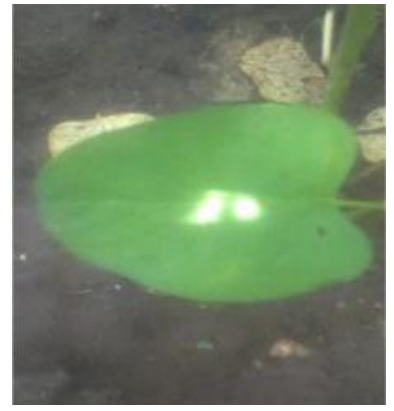

Fig.7 Cluster object1

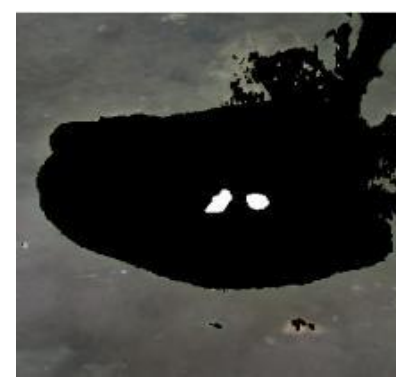

Fig.9 Cluster Object 2

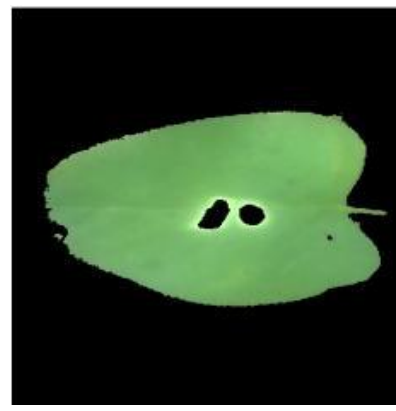

Fig 8.Double Image

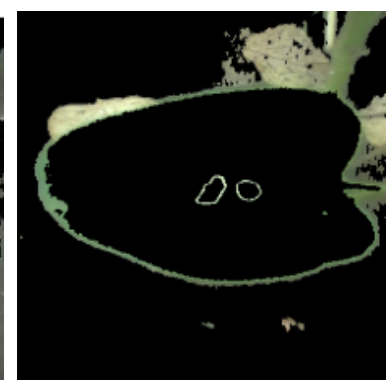

Fig 10. K -Mean Clustering Final Detection 


\section{CONCLUSION}

To detect disease in plant is one of the important research fields, as it decreases the production of agriculture. Many works has been proposed in this way. from this method we can identify the disease present in monocot or dicot. K-mean clustering method is the popular method which is also used in this article. List of references is also added to this article for more detailed description .This paper deals with the accuracy and fast approach of disease detection. The system will help the farmer to increase the production of agriculture.

\section{REFERENCES}

[1] Dubey S., Dixit P., Singh h N., Gupta N, “ infected Part Detection using K-Means Clustering Segmentation Technique" International Journal of Artificial Intelligence and Interactive Multimedia, Vol. 2, No. 2.

[2] Blasco J., Alexios N., Molto E., "Machine Vision System for automatic quality grading of fruitproceeding of science direct, Automation and Emerging Technologies, pp. 415-423, 2003.

[3] Pujara j ., Vakundimath R., Byadgi A "image processing based detection of fungai disease of plants" Proceedings of ELSEVIER, international conference on information and communication technologies(ICICT2014). pp. 1802 -1808, 2014.

[4] Monika Jhuria, Ashwani Kumar and Rushik esh Borse, "Image processing for smart farming detection of disease and fruit grading," IEEE 2nd International Conference on Image Information Processing (ICIIP), Shimla, pp 521$526,2013$.

[5] P.Revathi and M.Hemalatha, "Classification of Cotton Leaf Spot Diseases Using Image Processing Edge Detection Techniques," IEEE International Conference on Emerging Trends in Science, Engineering and Technology (INCOSET), Tiruchirappalli, pp 169-173, 2012.

[6] Dubey SR, Jalal AS. Adapted Approach for Fruit Disease Identification using Images. International Journal of Computer Vision and Image Processing. 2012;2(3):5165.

[7] Patil Sanjay B et al. Leaf disease severity measurement using image processing. Int J Eng Technol 2011; 3(5):297-301.

[8] Vijayaraghavan V, Garg A, Wong CH, Tai K. Estimation of mechanical properties of nanomaterials using artificial intelligence methods. Appl Phys A 2013:1-9.

[9] Ravi C.Shinde, Jibu Mathew $\mathrm{C}$ and Prof. C. Y. Patil"Segmentation Technique for Soybean Leaves Disease Detection" International Journal of Advanced Research (2015), Volume 3, Issue 5, 522-528

[10] Xiao-dan M, Hai-ou G, Fen T. Investigation on the Extraction of Soybean Brown Spot Based on Improved Genetic Algorithm. Information Science and Management Engineering. 2010;1:14 - 17. Xi'an: IEEE.

[11] Weizheng S, Yachun W, Zhanliang C, Wei H. Grading mathod of leaf spotInfection based image processing" International conference on comuter science and software engineering. 2008;491-494. Wuhan, Hube: IEEE. doi:10.1109/CSSE.2008.1649

[12] Ms. Chinki Chandhok, Mrs. Soni Chaturvedi, Dr. A. A.
Khurshid "An Approach to Image Segmentation Using K-means Clustering Algorithm" International Journal of Information Technology (IJIT) ,Volume-1,Issue1,August 2012, ISSN 2279-008X.

[13] Cui D, Zhang Q, Li M, Zhao Y, Hartman GL. Detection of soybean rust using a multispectral image sensor", Sens. \& Instrumen. Food Qual. 2009;3:49-56.

[14] Rabia Masood, S.A. Khan, M.N.A. Khan, Plants Disease Segmentation using Image Processing I.J. Modern Education and Computer Science, 2016, 1, 24-32

[15] Yinmao Song, Zhihua Diao, Yunpeng Wang, Huan Wang Image Feature Extraction of Crop Disease 2012 IEEE Symposium on Electrical \& Electronics Engineering (EEESYM). College of Electrical and Information Engineering Zhengzhou University of Light Industry.

[16] S. Arivazhagan, R. Newlin Shebiah*, S. Ananthi, S. Vishnu Varthini. Detection of unhealthy region of plant leaves and classification of plant leaf diseases using texture features. March, 2013 Agric Eng Int: CIGR Journal. Department of Electronics and Communication Engineering, Mepco Schlenk Engineering College, Sivakasi Tamilnadu, 626 005, India.

[17] N.Valliammai', S.N.Geethaiakshmi2. Multiple noise reduction using hybrid method for leaf recognition. $\mathrm{H}$. Al-Hiary, S. Bani-Ahmad, M. Reyalat, M. Braik and Z. Al Rahamneh. Jordan. Fast and Accurate Detection andClassification of Plant Diseases. International Journal of Computer Applications (0975 - 8887) Volume 17No.1, March 201. Department of Information Technology, Al-Balqa' Applied University, Salt Campus.

[18] Anup Vibhute Assistant Professor, BMIT, Solapur(India) and S K Bodhe Phd, Professor, Applications of Image Processing in Agriculture: A Survey. International Journal of Computer Applications (0975 - 8887) Volume 52- No.2, August 2012. College of Engineering, Pandharpur.

[19] Crop pest surveillance and advisory project (CROPSAP) in maharashtra (2011-12)

[20] Keyvan Asefpour Vakilian, Jafar Massah. Development and performance evaluation of a robot to early detection of nitrogen deficiency in greenhouse cucumber (cucumis sativus) with machine vision. International Journal of Agriculture: Research and Review. Department of Agrotechnology, College of Abouraihan, University of Tehran, Tehran, Iran.

[21] Mr. Jagan Bihari Padhy, Devarsiti Dillip Kumar, Ladi Manish and Lavanya Choudhry" Leaf Disease Detection Using K-Means Clustering And Fuzzy Logic Classifier"IJSET, Volume 02, No. 5, May 2016,23950900 .

[22] Abdallah A. Alshennawy, and Ayman A. Aly," Edge Detection in Digital Images Using Fuzzy Logic Technique", World Academy of Science, Engineering and Technology 272009.

[23] Sunita I. Naik, Vivekanandreddy, S. S. Sannakki" Plant Disease Diagnosis System for Improved Crop Yield", International Journal of Innovations in Engineering and Technology (IJIET), Vol. 4 Issue 1 June 2014, ISSN: $2319-1058$. 